\title{
Randomised clinical trial: Polyethylene glycol 3350 with sports drink vs. polyethylene glycol with electrolyte solution as purgatives for colonoscopy--the incidence of hyponatraemia.
}

\author{
Rebecca Matro \\ Thomas Jefferson University \\ Constantine Daskalakis \\ Thomas Jefferson University \\ Dan Negoianu \\ University of Pennsylvania \\ Follow this and additional works at: https://jdc.jefferson.edu/gastro_hepfp \\ Io Katz

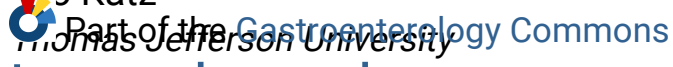 \\ Let us know how access to this document benefits you
}

Thomas Jefterson University

\section{Recommended Citation}

Matro, Rebecca; Daskalakis, Constantine; Negoianu, Dan; Katz, Leo; Henry, Cassandra; Share, Michael; and Kastenberg, David, "Randomised clinical trial: Polyethylene glycol 3350 with sports drink vs. polyethylene glycol with electrolyte solution as purgatives for colonoscopy--the incidence of hyponatraemia." (2014). Division of Gastroenterology and Hepatology Faculty Papers. Paper 32.

https://jdc.jefferson.edu/gastro_hepfp/32

This Article is brought to you for free and open access by the Jefferson Digital Commons. The Jefferson Digital Commons is a service of Thomas Jefferson University's Center for Teaching and Learning (CTL). The Commons is a showcase for Jefferson books and journals, peer-reviewed scholarly publications, unique historical collections from the University archives, and teaching tools. The Jefferson Digital Commons allows researchers and interested readers anywhere in the world to learn about and keep up to date with Jefferson scholarship. This article has been accepted for inclusion in Division of Gastroenterology and Hepatology Faculty Papers by an authorized administrator of the Jefferson Digital Commons. For more information, please contact: JeffersonDigitalCommons@jefferson.edu. 


\section{Authors}

Rebecca Matro, Constantine Daskalakis, Dan Negoianu, Leo Katz, Cassandra Henry, Michael Share, and David Kastenberg 
Title: Randomised clinical trial: Polyethylene glycol 3350 with sports drink versus polyethylene glycol with electrolyte solution as purgatives for colonoscopy - the incidence of hyponatremia

Short title: PEG-SD vs. PEG-ELS and hyponatremia

Authors: Rebecca Matro M.D. ${ }^{1}$, Constantine Daskalakis Sc.D ${ }^{2}$, Daniel Negoianu M.D.*33, Leo Katz M.D. ${ }^{1}$, Christopher Henry, M.D. ${ }^{4}$, Michael Share M.D.*5 , David Kastenberg, M.D. ${ }^{1}$

1. Thomas Jefferson University Hospital, Division of Gastroenterology and Hepatology; 2. Thomas Jefferson University, Division of Biostatistics; 3. Hospital of the University of Pennsylvania, Renal, Electrolyte and Hypertension Division; 4. Thomas Jefferson University Hospital, Department of Internal Medicine; 5. Ronald Reagan UCLA Medical Center, Department of Internal Medicine

*These two authors both worked at TJUH during the initial phases of the study. They moved to other institutions prior to manuscript submission.

Keywords: purgative, colonoscopy, hyponatremia

\section{Corresponding Author:}

David Kastenberg, M.D.

David.Kastenberg@jefferson.edu

132 S. $10^{\text {th }}$ Street

480 Main Building

Philadelphia, PA 19107

Phone: 215-955-8900

Fax: 215-503-2578 


\section{Abstract}

Background: Polyethylene glycol 3350 plus sports drink (PEG-SD) is a hypoosmotic purgative commonly used for colonoscopy, though little safety data is available.

Aim: The purpose was to evaluate the effect of PEG-SD on serum sodium (Na) and other electrolytes compared with polyethylene glycol 3350-electrolyte solution (PEG-ELS).

Methods: We performed a single center, prospective, randomized, investigator-blind study comparison of PEG-ELS to PEG-SD in outpatients undergoing colonoscopy. Laboratory studies were obtained at baseline and repeated immediately before and after colonoscopy. The primary endpoint was development of hyponatremia $(\mathrm{Na}<135 \mathrm{mmol} / \mathrm{L})$ the day of colonoscopy. Changes in levels of electrolytes were computed as the difference between the lowest value on the day of colonoscopy and baseline. Purgative tolerance and cleansing efficacy were assessed.

Results: 389 patients were randomized, and 364 took purgative and had baseline and day of colonoscopy labs (180 PEG-SD, 184 PEG-ELS). The groups were well matched except for a higher fraction of women and blacks in PEG-ELS group. 7 patients (3.9\%) in PEG-SD and 4 patients $(2.2 \%)$ in PEG-ELS developed hyponatremia (OR=1.82, 95\% CI: 0.45 to 8.62, $\mathrm{p}=0.376$ ). Changes in electrolytes from baseline were small but significantly worse with PEGSD for sodium $(\mathrm{Na})$, potassium $(\mathrm{K})$, and chloride $(\mathrm{Cl})(\mathrm{p}=0.001,0.012$, and 0.001 , respectively). Preparation completion, adverse events, and overall colon cleansing were similar between the groups, but there were more excellent preparations with PEG-ELS (52\% vs. 30\%; p=0.001). Conclusions: Greater, but very modest, electrolyte changes occur with PEG-SD. Hyponatremia is infrequent with PEG-SD and PEG-ELS. A significant increase in hyponatremia was not identified for PEG-SD vs. PEG-ELS, but the sample size may have been inadequate to identify a small, but clinically important, difference. ClinicalTrials.gov identifier NCT01299779. 


\section{Introduction}

Over 14 million colonoscopies are performed in the United States every year and as many as $50 \%$ of patients use over-the-counter bowel purgatives. ${ }^{1}$ One such popular purgative is polyethylene glycol 3350 combined with sports drink (PEG-SD), often used in conjunction with bisacodyl. PEG-SD is hypoosmotic, containing substantially less sodium, potassium, and chloride compared to polyethylene glycol-electrolyte (PEG-ELS) formulations available by prescription. ${ }^{2-6}$ PEG-ELS is nearly isosmotic to minimize electrolyte shifts and replace those lost during purgation.

Published studies have evaluated the efficacy of PEG-SD, but little data exists regarding its safety or risk of electrolyte abnormalities. In particular, a hypoosmotic purgative in patients following a clear liquid diet may increase the risk for hyponatremia. Hypoosmolar hyponatremia may occur with excessive ingestion of electrolyte-free water - typically due to water retention by the kidneys in response to antidiuretic hormone $(\mathrm{ADH}) . \mathrm{ADH}$ is released in the setting of intravascular volume depletion, such as with bowel purgation. Hyponatremia also occurs in euvolemic patients, most commonly due to the syndrome of inappropriate ADH (SIADH). SIADH is associated with many factors including nausea, anxiety, pain, trauma, tumors, and certain medications. ${ }^{7}$ Many of these factors, and dehydration, are seen in patients coming for colonoscopy. Severe hyponatremia may result in seizures, arrhythmias, coma, and death.

The incidence of hyponatremia associated with polyethylene based purgatives is difficult to estimate since neither pre- nor post-colonoscopy chemistries are routinely performed. Hyponatremia does occur with PEG-ELS. In a prospective study of 40 patients undergoing colonoscopy after 2-3L PEG-ELS, the incidence of hyponatremia was $7.5 \%{ }^{8}$ Ayus et al. described two patients with severe hyponatremia after consuming 4L PEG-ELS, one of whom 
developed seizures $(\mathrm{Na}=116 \mathrm{mmol} / \mathrm{L})$ and the other died from cardiac arrest $(\mathrm{Na}=122$ mmol/L). ${ }^{9}$ A larger prospective trial comparing $2 \mathrm{~L}$ to $4 \mathrm{~L}$ PEG-ELS demonstrated a 5-6\% incidence of new-onset peri-procedure hyponatremia in both groups. ${ }^{10}$

Hyponatremia has also been reported with PEG-SD. A case report described new onset seizure after taking PEG-SD. ${ }^{11}$ In a case series reported only in abstract, nine patients who underwent outpatient colonoscopy developed hyponatremia. ${ }^{12}$ Eight were hospitalized and two of those had serious sequelae. Finally, in a recent study comparing the efficacy of multiple regimens of PEG-ELS and PEG-SD that was not powered for the outcome of hyponatremia, no instances of hyponatremia were observed. ${ }^{13}$

The primary objective of this study was to evaluate whether the incidence of hyponatremia is greater with PEG-SD compared to PEG-ELS. We hypothesized that compared to PEG-SD, hyponatremia would occur significantly less often with PEG-ELS.

\section{Materials and Methods}

\section{Participants}

This was a prospective, randomized, investigator blind study at a single academic center, Thomas Jefferson University Hospital (TJUH). The study was approved by the TJUH institutional review board (IRB) and registered at ClinicalTrials.gov, with identifier NCT01299779. All patients provided informed consent. Patients aged $\geq 18$ years undergoing elective outpatient colonoscopy were eligible to participate. Exclusion criteria included history of hyponatremia, prior difficulty with phlebotomy, end stage renal disease on hemodialysis, recent myocardial infarction ( $<3$ months) or unstable angina, bowel obstruction, greater than $50 \%$ colon resection, pregnancy, breast feeding, moderate or severe psychiatric illness, or inability to 
provide informed consent. Initially, patients with New York Heart Association Congestive Heart Failure Class 3 or 4, Stage 4 or 5 chronic renal insufficiency (GFR <30), and decompensated liver disease (ascites, recent variceal bleed) were excluded. However, after $\sim 25 \%$ enrollment (101 patients), an amendment was approved by the IRB to include patients with any degree of congestive heart failure or liver disease, and patients with renal insufficiency except for those on dialysis. Although these patients are routinely excluded from purgative studies performed for the purpose of seeking FDA approval ${ }^{10,14-17}$, we wanted to include patients representative of the outpatient setting who might be at risk of hyponatremia.

\section{Study Design}

Once enrolled, patients underwent baseline assessment 2-5 business days before colonoscopy during which patients provided informed consent. Demographic and clinical data were collected including age, race, medical and surgical history, medications, indication for colonoscopy, height, weight, blood pressure, pulse, respiratory rate, temperature and orthostatic vital signs. Blood ("baseline”) was obtained for comprehensive metabolic panel, glucose, serum osmolality, and serum ADH. Patients were subsequently excluded if baseline laboratory values were abnormal as follows: sodium $<135 \mathrm{mmol} / \mathrm{L}$ or $>146 \mathrm{mmol} / \mathrm{L}$, potassium $<3.3 \mathrm{mmol} / \mathrm{L}$ or $>5.5 \mathrm{mmol} / \mathrm{L}$, or calcium $<8.0 \mathrm{mg} / \mathrm{dL}$ or $>11.0 \mathrm{mg} / \mathrm{dL}$. When patients were excluded for abnormal baseline laboratory values, the patients' physicians were notified to dictate further care.

Using a randomization schedule generated by the website http://www.randomization.com, patients were assigned to take either 2L PEG-ELS or 2L PEGSD and bisacodyl by a research coordinator not involved in performing the colonoscopy. Patients were provided verbal and written instructions regarding purgative preparation, dosing, and diet. 
Patients receiving PEG-ELS were provided with a standard kit (Moviprep®, Salix Pharmaceuticals, Morrisville, NC), and consumed 1L PEG-ELS plus 500cc clear liquids at 6 pm the night prior and again four hours prior to colonoscopy. The total PEG-ELS dose contains $200 \mathrm{~g}$ PEG 3350, 15g sodium sulfate, $5.382 \mathrm{~g}$ sodium chloride, $2.03 \mathrm{~g}$ potassium chloride, $9.4 \mathrm{~g}$ ascorbic acid, and 11.8g sodium ascorbate, plus lemon flavoring, aspartame and acesulfame potassium sweeteners. ${ }^{3}$ When reconstituted in $2 \mathrm{~L}$ water, PEG-ELS is hyperosmolar at $420 \mathrm{mOsm} / \mathrm{L}$. Those assigned to PEG-SD received two $5 \mathrm{mg}$ tablets of bisacodyl, two $119 \mathrm{~g}$ bottles of PEG 3350 (Miralax®, Merck \& Co., Inc., Whitehouse Station, NJ) and two 32 ounce bottles of Lemon Lime flavored sports drink (Gatorade G Series 2®, PepsiCo, Inc., Chicago, IL). The PEG-SD group took $10 \mathrm{mg}$ of bisacodyl at $3 \mathrm{pm}$ the day prior. One liter of sports drink was mixed with one $119 \mathrm{~g}$ bottle PEG 3350 at $6 \mathrm{pm}$ the night prior and drunk over 1 hour (8 ounces every 15 minutes), and this was repeated four hours before colonoscopy. Each 1L of sports drink contains approximately $20 \mathrm{mEq} / \mathrm{L}$ sodium, $3 \mathrm{mEq} / \mathrm{L}$ potassium, $12 \mathrm{mEq} / \mathrm{L}$ chloride, $12 \mathrm{mmol} / \mathrm{L}$ citrate, and $3 \mathrm{mmol} / \mathrm{L}$ phosphate, and the osmolality is approximately $360 \mathrm{mOsm} / \mathrm{L} .{ }^{18-21}$ Much of this is carbohydrate, which is metabolized rapidly. Sports drink is therefore quite hypotonic, with a cationic electrolyte concentration of approximately $23 \mathrm{mEq} / \mathrm{L}$.

Diet was standardized and identical for both groups with a low residue breakfast before 10 am the day prior, followed by clear liquids up to 2.5 hours before colonoscopy. In addition to the purgative requirements, patients were instructed to consume at least an additional 4L of clears. A research physician uninvolved in the colonoscopy was available to patients by phone at any time.

Immediately prior to colonoscopy, patients completed questionnaires evaluating compliance, tolerance, fluid ingestion, and anxiety. Compliance was assessed by whether $<90 \%$ 
or $\geq 90 \%$ of each purgative dose was ingested. Tolerance was measured using a 10-point Likert scale which rated nausea, vomiting, abdominal pain, lightheadedness, and bloating from 0 (none) to 10 (severe). In addition to the $2 \mathrm{~L}$ purgative, patients estimated fluid intake the day prior to colonoscopy as <3L, 3-5L, or $>5 \mathrm{~L}$ (see "Patient Questionnaire" Addendum). Anxiety was scored using the Beck Anxiety Inventory@ (BAI), consisting of 21 items rated on a scale of 0 (none) to 3 (severe) and added into a final score: minimal (0-7), mild (8-15), moderate (16-25), and severe (26-63). ${ }^{22}$ Subjects' weights and vital signs, including orthostatic assessments, were measured. Prior to placement of an intravenous (IV) line, blood was drawn ("Pre"). Patients received 1L of Plasma-lyte (Plasma-lyte®, Baxter International Inc., Deerfield, IL), (each 100 mL contains 526mg Sodium Chloride, 502mg Sodium Gluconate, 368mg Sodium Acetate Trihydrate, 37mg of Potassium Chloride, and 30mg of Magnesium Chloride) in the peri-procedure period.

During the procedure, an investigator documented extent of exam, adverse events and interventions. After the procedure, the endoscopist graded the preparation using the Aronchick Scale for the whole colon and right colon, with excellent or good considered adequate and fair or poor considered inadequate. ${ }^{23}$ Patients received monitored anesthesia care with propofol-based sedation administered by a certified registered nurse anesthetist. Following the colonoscopy and 1L of Plasma-lyte, blood ("Post") was again collected. An investigator uninvolved in the colonoscopy reviewed the final pathology report and this was used to record polyp size and histology.

Patients were asked not to discuss their preparation with the endoscopist at any time. All instructions and assessments were done privately without the endoscopist present. At the time of colonoscopy, the endoscopist documented whether s/he remained blind to the preparation. All colonoscopies were performed by attending gastroenterologists without fellow participation. 


\section{Outcome Measures}

The primary outcome was the development of hyponatremia on the day of colonoscopy (serum sodium $<135 \mathrm{mmol} / \mathrm{L}$ at pre- or post-colonoscopy assessment). Secondary endpoints included the change from baseline for serum electrolytes (sodium, potassium, chloride, calcium corrected for albumin), renal function, serum osmolality, and vasopressin. Additional secondary outcomes included the development of abnormal serum electrolyte values, development of orthostatic hypotension, side effects, compliance with preparation completion, and colonoscopy quality (preparation, completion, adenoma detection). Changes in levels of electrolytes and renal function were computed as the difference between the lowest value on the day of colonoscopy (pre- or post-procedure) and the baseline assessment. Changes in creatinine, BUN, osmolality, and vasopressin were computed as the difference between the highest value on the day of colonoscopy and the baseline assessment. Using a 2-sided chi-squared test with alpha 0.05 and a target sample size of 185 patients per arm, the study was designed to have at least $80 \%$ power to detect a threefold difference (odds ratio of 0.33 ) between PEG-ELS and PEG-SD with respect to the incidence of hyponatremia (5\% vs. $14 \%)$.

\section{Statistical Analysis}

Exact methods (exact confidence interval and Fisher's exact test) were used to compare the two groups (PEG-ELS vs. PEG-SD) on incidence of hyponatremia and other electrolyte abnormalities, preparation completion and quality, incidence of side effects, and colonoscopy findings. Student's t-test was used to compare the two groups on the change in serum measures between baseline and day of colonoscopy, and the Kruskal-Wallis test was used to compare the two groups on the preparation's side effects and anxiety scores. All analyses followed the intentto-treat principle, but ineligible patients or those who withdrew after randomization were 
excluded from all analyses. In addition, patients who did not have blood work were excluded from the electrolyte analyses. All analyses were conducted in SAS 9.3 (SAS Institute Inc., Cary, NC) and StatXact 9 (Cytel Software Corp., Cambridge, MA). All authors had access to the final study data and approved the manuscript for publication.

\section{Results}

From June 2010 through June 2012, 638 patients were assessed for eligibility and 389 were randomized to PEG-ELS or PEG-SD (figure 1). Twenty-five patients were excluded, including 16 patients for abnormal baseline laboratories (13 with hyponatremia, range 130-134 mmol/L). The analyses included 364 patients, 180 in the PEG-SD group and 184 in the PEGELS group. The study colonoscopies were performed by nine physicians, although $>75 \%$ were performed by two endoscopists. Endoscopist masking was preserved for 175 patients in each group ( $\mathrm{p}=0.415$ ). Table 1 summarizes study patient characteristics. Compared to the PEG-SD group, the PEG-ELS group included more females and Blacks.

Phlebotomy was completed pre-procedure for all patients except one in the PEG-SD group, and post-procedure for 175 (97\%) in the PEG-SD group and $180(98 \%)$ in the PEG-ELS group. Hyponatremia was observed in 11 patients, 7 (3.9\%) in the PEG-SD group and 4 (2.2\%) in the PEG-ELS group (odds ratio, $\mathrm{OR}=1.82$, exact $95 \%$ confidence interval, CI: 0.45 to 8.62 , $\mathrm{p}=0.376$ ). For these 11 patients, the mean change in sodium was $-5.3, \mathrm{SD}=2.7$, with no instances of $\mathrm{Na}<131 \mathrm{mmol} / \mathrm{L}$. Table 2 compares the 11 patients who developed hyponatremia in both groups with the 353 patients who did not. Nine of the 11 patients $(82 \%)$ who developed hyponatremia were taking a diuretic (thiazide, loop, or potassium-sparing) at the time of colonoscopy, compared to 52 of the $353(15 \%)$ of patients without hyponatremia $(\mathrm{p}=0.001)$. 
Anxiety scores were similarly low in patients who developed hyponatremia and those who did not $($ mean $=3.0$ vs. 3.8, respectively).

Table 3 summarizes the incidence of serum electrolytes outside the normal range at both baseline and day of colonoscopy. For all electrolytes, serum levels outside the normal range on the day of colonoscopy were not significantly greater in the PEG-SD group. Four patients had an elevated creatinine $(>1.4 \mathrm{mg} / \mathrm{dL})$ on the day of colonoscopy, $3(0.7 \%)$ in the PEG-SD group and $1(0.5 \%)$ in the PEG-ELS group $(\mathrm{p}=0.368$, range $1.5-1.7 \mathrm{mg} / \mathrm{dL})$. In 2 of these PEG-SD patients, the creatinine elevation was new.

Table 4 summarizes the serum levels of electrolytes at baseline and day of colonoscopy. Sodium levels decreased by an average of $0.7 \mathrm{mmol} / \mathrm{L}$ in the PEG-SD group and increased by an average of $0.5 \mathrm{mmol} / \mathrm{L}$ in the PEG-ELS group $(\mathrm{p}=0.001)$. The decrease from baseline was also small but statistically greater for potassium and chloride in the PEG-SD group. Although hypokalemia was somewhat more common in the PEG-ELS group, the degree of hypokalemia was greater among those in the PEG-SD group. For calcium, creatinine, or osmolality, the two groups were not significantly different.

Orthostatic hypotension was rare and not statistically different between the two groups $(\mathrm{p}=0.677)$. At baseline, there were 3 cases of orthostatic hypotension in the PEG-SD group and 8 in the PEG-ELS group. On the day of colonoscopy, there were 6 and 8 patients, respectively, and in no case did hypotension occur in the same patient at both time points. No patients with orthostatic hypotension were symptomatic. There was no significant difference between study groups in change in vital signs from baseline to colonoscopy.

Purgative completion, fluid intake, side effects and colonoscopy findings are summarized in Table 5. Completion of $\geq 90 \%$ of both purgative doses was high for all study patients $-98 \%$ 
for PEG-SD and 95\% for PEG-ELS. Most patients consumed 3-5 liters of fluids the day before colonoscopy. Overall fluid consumption was greater in the PEG-SD group ( $\mathrm{p}=0.007)$. Neither incidence nor severity of any side effect differed significantly between the two groups $(\mathrm{p}=0.124)$, although more patients receiving PEG-SD experienced nausea $(\mathrm{p}=0.061)$. Levels of anxiety were low and similar between the two study groups $(\mathrm{p}=0.162)$. Minimal anxiety was reported by $86 \%$ of the PEG-SD group vs. $84 \%$ of the PEG-ELS patients.

The average number of polyps per patient was 1.0 in both groups $(p=0.962)$. In the PEGSD group, 118 adenomas were detected in 179 patients ( 0.66 adenomas/patient), and 57 patients had at least one adenoma (32\%). In the PEG-ELS group, 125 adenomas were detected in 183 patients (0.68 adenomas/patient), and 59 patients had at least one adenoma (32\%). The two groups did not differ on the histology of the 356 polyps $(\mathrm{p}=0.982)$, having similar fractions of tubular adenomas (59\% in PEG-SD, 56\% in PEG-ELS), sessile serrated adenomas (8\% in both groups), traditional serrated adenomas (3\% in both groups), and hyperplastic polyps (30\% and $33 \%$, respectively).

Two serious adverse events occurred during the study. After taking the preparation but before colonoscopy, one patient in the PEG-SD group had an acute myocardial infarction requiring cardiac catheterization and subsequent coronary bypass surgery. One patient in the PEG-ELS group had an asthma attack after taking 1L of the preparation and did not undergo colonoscopy.

Figure 2 illustrates preparation quality. A similar fraction of patients in the two study groups had adequate preparations (whole colon: 87\% PEG-SD, 86\% PEG-ELS, $\mathrm{p}=0.878$; right colon: $85 \%$ PEG-SD, 88\% PEG-ELS, p=0.539). However, the PEG-ELS group had a greater 
proportion of excellent preparations (whole colon: PEG-SD, 57\% good, 30\% excellent; PEGELS, 34\% good, 52\% excellent; $\mathrm{p}=0.001)$.

\section{Discussion}

PEG-SD is commonly prescribed for bowel preparation. No clinical trials have previously evaluated safety endpoints, such as electrolyte and volume changes after administration, as a primary outcome. A randomized study powered for efficacy, but not safety, compared split dosing to day prior dosing of PEG-SD in 114 patients. ${ }^{13}$ Serum chemistries drawn immediately prior to colonoscopy showed no cases of hyponatremia. Nevertheless, case reports of patients taking PEG-ELS or PEG-SD indicate that severe hyponatremia does occur. ${ }^{8-9,11-12}$ The reported incidence rates of any degree of hyponatremia following PEG-ELS may be as high as 8\%. ${ }^{9-10}$ Because PEG-SD is markedly more hypo-osmotic than PEG-ELS, the purpose of this study was to determine whether PEG-SD confers a greater risk for hyponatremia.

This large, prospective, randomized study showed that hyponatremia occurs very infrequently following ingestion of either PEG-ELS or PEG-SD. There was no significant difference in the incidence of hyponatremia between these purgatives. However, the observed incidence of hyponatremia with both purgatives was low and less than predicted. Using the observed incidence, a study four times larger would be needed to prove hyponatremia occurs significantly less often with PEG-ELS than with PEG-SD using the pre-set margins. Furthermore, when hyponatremia occurred, the degree was minor (range 131-134 mmol/L) and asymptomatic. The incidence of hypokalemia, hypochloremia, and hypocalcemia was also uncommon and did not significantly differ between the two study groups. A total of 13 patients (6 PEG-SD, 7 PEG-ELS) had some degree of hemolysis on their day of colonoscopy labs and were excluded from the potassium analyses; some of these patients could have been hypokalemic 
as well. One patient in the PEG-SD group who had hyponatremia both pre- and postcolonoscopy had mild hemolysis on post-colonoscopy blood draw.

Among study patients, there was a significantly greater decrease in serum sodium in the PEG-SD group, although this reduction was very modest. Similarly, small but significantly greater reductions in serum potassium and calcium were also observed in the PEG-SD group.

Differences in medication use and medical conditions were observed between patients who developed hyponatremia and those who did not. Among study patients developing hyponatremia, diuretic use was significantly greater and the use of an ACEI or ARB more common. Significantly more patients who developed hyponatremia had diabetes, and a greater proportion had hypertension, cardiovascular disease, or liver disease. Similar characteristics were observed among the 13 patients excluded from this study for hyponatremia at baseline screening - diuretic use in 6, ACEI or ARB use in 3, cardiovascular disease in 8, diabetes in 4, and liver disease in 4 .

Adverse events, compliance, and colonoscopy quality measures were similar in patients receiving PEG-ELS and PEG-SD. The two groups were similar at baseline and on the day of colonoscopy with respect to vital signs, changes in vital signs, and the incidence of orthostatic hypotension. No patients exhibited symptoms or signs of volume depletion. The incidence of adverse events was not significantly different between the two groups, and nearly all patients in both groups completed both doses of purgative. Finally, similar rates of adenoma detection and colonoscopy completion were observed between the study groups.

Bowel cleansing was evaluated as a secondary endpoint. Overall, the quality of bowel preparation was similar between the two groups, with no difference in the number of patients with adequate or inadequate preparations. However, focusing on adequate preparations, which 
were categorized as excellent or good, excellent bowel preparations were observed in significantly more patients in the PEG-ELS group.

There are several strengths to our study. Prior studies evaluating PEG-SD have had cleansing efficacy as the primary endpoint, including a publication reporting on the incidence of hyponatremia but not powered to evaluate this outcome. ${ }^{13}$ Our randomized controlled trial was designed specifically to evaluate safety measures, with the primary endpoint the incidence of hyponatremia. Other serum electrolytes, renal function, and volume status were also studied. All study patients followed a split dosing schedule, and both groups were consistent with respect to the timing of purgative ingestion (pre-colonoscopy) and completion of peri-procedure fluids (post-colonoscopy).

The population studied is fairly representative of outpatients reporting for colonoscopy with respect to age, gender, indications and medical history. The procedure for anesthesia was also standardized. We controlled for anxiety, as this is an independent factor associated with hyponatremia via activation of the hypothalamus-pituitary-adrenal (HPA) axis and increased levels of vasopressin. ${ }^{24,25}$ Furthermore, the volume of fluids ingested the day prior was also considered in the analysis, as ingestion of free water increases the risk for hyponatremia.

There are several limitations to our study. First, although our primary study endpoint was serum sodium $<135 \mathrm{mmol} / \mathrm{L}$, serious adverse events related to hyponatremia such as seizure, coma, arrhythmias, and even death are usually associated with serum sodium $<130 \mathrm{mmol} / \mathrm{L}$. This study was not designed to detect the incidence of severe hyponatremia. A much larger study with thousands of patients would be needed to assess the incidence of severe hyponatremia is significantly different between PEG-SD and PEG-ELS. Second, the much lower use of diuretics in the PEG-SD group may have had a mitigating effect on the incidence of hyponatremia in these 
patients as compared to the PEG-ELS group. Unfortunately, because of the small number of hyponatremia cases, multivariable analyses that would have adjusted for baseline differences were not possible. Third, few patients were actually enrolled with medical conditions placing them at higher risk for hyponatremia, such as those with chronic liver or kidney disease. Heart failure was classified within the category of cardiovascular disease. It is worthwhile noting that vulnerable patient populations such as those with cirrhosis, heart failure, and renal disease are standardly excluded from clinical purgative trials performed for the purpose of gaining FDA approval. $^{10,14-17}$

Unlike routine clinical practice, baseline serum electrolytes were required for this study, and those found to be hyponatremic were excluded. These patients did not have a history of hyponatremia and, in all likelihood, would have undergone colonoscopy in a non-study setting. The follow up of these patients is not available as they were discharged to the care of their primary gastroenterologist. The inclusion of such patients may have increased the incidence of hyponatremia for both study groups.

In conclusion, this study shows that hyponatremia is a rare event after ingestion of either PEG-ELS or PEG-SD for colonoscopy. Patients using diuretics may be more likely to develop hyponatremia in the setting of purgative ingestion for colonoscopy. While the risk associated with specific medications or medical disorders deserves further investigation, PEG-SD is a reasonable option for patients without a history of hyponatremia or medical conditions conferring a high risk. Although this study does not prove the safety of PEG-SD for use as a bowel purgative, it suggests that in patients at low risk for hyponatremia, the risk for hyponatremia with either PEG-SD or PEG-ELS for colonoscopy is small. 


\section{ACKNOWLEDGMENTS}

We wish to thank Angela Gordon, RN for her assistance with patient visits and procedures and laboratory and data collection. We also thank Irene Dialino for her assistance with data collection during procedures, and subject chart organization and management. Finally, we acknowledge Cynthia Miller, RN for research assistance and creating the study database.

\section{STATEMENTS OF INTEREST}

1. Author's declaration of personal interests

a. David Kastenberg receives research support/grants and serves as a consultant and advisory board member for Salix Pharmaceuticals, Inc.

2. Declaration of funding interests

a. This study was funded by Salix Pharmaceuticals, Inc., who provided funding for all study medications, including PEG-ELS, PEG 3350, sports drink, and bisacodyl.

b. Salix Pharmaceuticals, Inc. was NOT involved in the statistical design, data analysis, or writing of the manuscript. 


\section{References}

1. Seeff LC, Richards TB, Shapiro JA, et al. How many endoscopies are performed for colorectal cancer screening? Results from CDC's survey of endoscopic capacity. Gastroenterol. 2004;127:1670-7.

2. Product Information for MiraLAX (Polyethylene Glycol 3350), Schering-Plough HealthCare Products, Inc.

3. Product Information for MoviPrep® (PEG-3350, sodium sulfate, sodium chloride, potassium chloride, sodium ascorbate and ascorbic acid for oral solution), Salix Pharmaceuticals, Inc.

4. Product Information for HalfLytely® (PEG-3350, sodium chloride, sodium bicarbonate and potassium chloride for oral solution and bisacodyl delayed release tablets). Braintree, MA: Braintree Laboratories, Inc.

5. Product Information for NuLYTELY (PEG-3350, Sodium Chloride, Sodium Bicarbonate and Potassium Chloride for Oral Solution), Braintree Laboratories, Inc.

6. Product Information for GoLYTELY (PEG-3350 and Electrolytes for Oral solution), Braintree Laboratories, Inc.

7. Ellison DH, Berl T. Clinical practice. The syndrome of inappropriate antidiuresis. N Engl J Med 2007; 356:2064.

8. Cohen CD, Keuneke C, Schiewann U, et al. Hyponatremia as a complication of colonoscopy. The Lancet. 2001;357:282-3. 
9. Ayus JC, Levine R, Arieff AI. Fatal dysnatraemia caused by elective colonoscopy. BMJ. 2003;326:328-4.

10. Ell C, Fischbach W, Bronisch JH, et al. Randomized trial of low-volume PEG solution versus standard PEG + Electrolytes for bowel cleansing before colonoscopy. Am J Gastroenterol. 2007;102:1-11.

11. Nagler J, Poppers D, Turetz M. Severe hyponatremia and seizure following a polyethylene-glycol based bowel preparation for colonoscopy. J Clin Gastroenterol. 2006;40:558-9.

12. Lewis JJ, Eswaran SL, Schoenfeld PS. Severe Hyponatremia associated with MiraLAXGatorade bowel prep prior to colonoscopy. Gastroenterol. 2011;140:S-18. [abstract]

13. Samarasena JB, Muthusamy R, Jamal MM. Split-Dosed MiraLAX/Gatorade Is an Effective, Safe, and Tolerable Option for Bowel Preparation in Low-Risk Patients: A Randomized Controlled Study. Am J Gastroenterol. 2012;107:1036-42.

14. Rex DK, Di Palma JA, Rodriguez R, McGowan J, Cleveland M. A randomized clinical study comparing reduced-volume oral sulfate solution with standard 4-liter sulfate-free electrolyte lavage solution as preparation for colonoscopy. Gastrointest Endosc. 2010;72:328-336.

15. Malik P, Balaban DH, Thompson WO, Galt DJ. Randomized study comparing two regimens of oral sodium phosphates solution versus low-dose polyethylene glycol and bisacodyl. Dig Dis Sci. 2009;54:833-841.

16. Rex D, Katz P, Bertiger G, et al. Split-dose administration of a dual-action, low-volume bowel cleanser for colonoscopy: the SEE CLEAR I study. Gastrointest Endosc. 2013;78:132-41. 
17. Katz P, Rex D, Epstein M, et al. A dual-action, low-volume bowel cleanser administered the day before colonoscopy: Results from the SEE CLEAR II Study. Am J Gastroenterol. 2013;108:401-9.

18. Drossman H. Chemical speciation analysis of sports drinks by acid-base titrimetry and ion chromatography: A challenging beverage formulation project. J Chem Ed. 2007;84: 124-7.

19. Goodman JW, Asplin JR, Goldfarb DS. Effects of two sports drinks on urinary lithogenicity. Urol Res. 2009;37: 41-6.

20. Rao SS, Summers RW, Rao GR, et al. Oral rehydration for viral gastroenteritis in adults: A randomized, controlled trial of 3 solutions. J Parenter Enteral Nutr. 2006;30:433-9.

21. http://gatorade.com.au/faq.php\#. Accessed December 29, 2013.

22. Beck AT, Epstein N, Brown G, Steer RA. An inventory for measuring clinical anxiety: Psychometric properties. J Consulting and Clinical Psychology. 1988;56:893-7.

23. Aronchick C, Lipshutz W, Wright S, et al. Validation of an instrument to assess colon cleansing. Am J Gastroenterol. 1999;94:2667. [abstract]

24. Surget A and Belzung C. Involvement of vasopressin in affective disorders. European J Pharmacology. 2008;583:340-9.

25. Zimmerman U, Spring K, Wittchen HU, et al. Arginine vasopressin and adrenocorticotropin secretion in response to psychosocial stress is attenuated by ethanol in sons of alcohol-dependent fathers. J Psychiatric Research. 2004;38:385-93. 
Table 1. Patient characteristics.

\begin{tabular}{|c|c|c|}
\hline & $\begin{array}{c}\text { PEG-SD } \\
(\mathrm{N}=180)\end{array}$ & $\begin{array}{c}\text { PEG-ELS } \\
(\mathrm{N}=184)\end{array}$ \\
\hline Age (years), mean (sd) & $55(11)$ & $56(10)$ \\
\hline \multicolumn{3}{|l|}{$\operatorname{Sex}, \mathrm{n}(\%)$} \\
\hline Male & $96(53)$ & $76(41)$ \\
\hline Female & $84(47)$ & $108(59)$ \\
\hline \multicolumn{3}{|l|}{ Race, n (\%) } \\
\hline White & $126(70)$ & $107(58)$ \\
\hline Black & $45(25)$ & $69(38)$ \\
\hline Other & $9(5)$ & $8 \quad(4)$ \\
\hline \multicolumn{3}{|l|}{ Indication, $\mathrm{n}(\%)$} \\
\hline Screening & $91(51)$ & $91(49)$ \\
\hline Surveillance & $32(18)$ & $39(21)$ \\
\hline Symptoms & $57(32)$ & $54(29)$ \\
\hline BMI $(\mathrm{kg} / \mathrm{m} 2),{ }^{*}$ mean $(\mathrm{sd})$ & $28.6(6.2)$ & $29.8(7.1)$ \\
\hline \multicolumn{3}{|l|}{ Past Medical History, ${ }^{* *} \mathrm{n}(\%)$} \\
\hline Cardiovascular disease or hypertension & $90(50)$ & $88(48)$ \\
\hline Hyperlipidemia & $60(33)$ & $50(27)$ \\
\hline GERD & $41(23)$ & $29(16)$ \\
\hline Psychiatric conditions & $35(19)$ & $27(15)$ \\
\hline Diabetes & $23(13)$ & $26(14)$ \\
\hline Pulmonary & $20(11)$ & $21(11)$ \\
\hline Endocrine (excluding diabetes) & $16(9)$ & $23(13)$ \\
\hline \multicolumn{3}{|l|}{ Medications, $* *$ n $(\%)$} \\
\hline Diuretics & $24(13)$ & $37(20)$ \\
\hline Loop & $0 \quad(0)$ & $11(6)$ \\
\hline Thiazide & $23(13)$ & $25(14)$ \\
\hline Potassium sparing & $1(1)$ & $6(3)$ \\
\hline $\begin{array}{l}\text { Angiotensin-converting enzyme inhibitors } \\
\text { or angiotensin receptor blockers }\end{array}$ & $49(27)$ & $49(27)$ \\
\hline Selective serotonin reuptake inhibitors & $23(13)$ & $14(8)$ \\
\hline Carbamazepine/Antiepileptics & $3(2)$ & $3(2)$ \\
\hline Proton pump inhibitors & $36(20)$ & $34(18)$ \\
\hline
\end{tabular}

SD: standard deviation. GERD: gastroesophageal reflux disease.

(*) Missing for 1 PEG-SD patient.

(**) Multiple medical conditions and medications could be reported. 
Table 2. Comparison of patients who developed hyponatremia with those who did not develop hyponatremia.

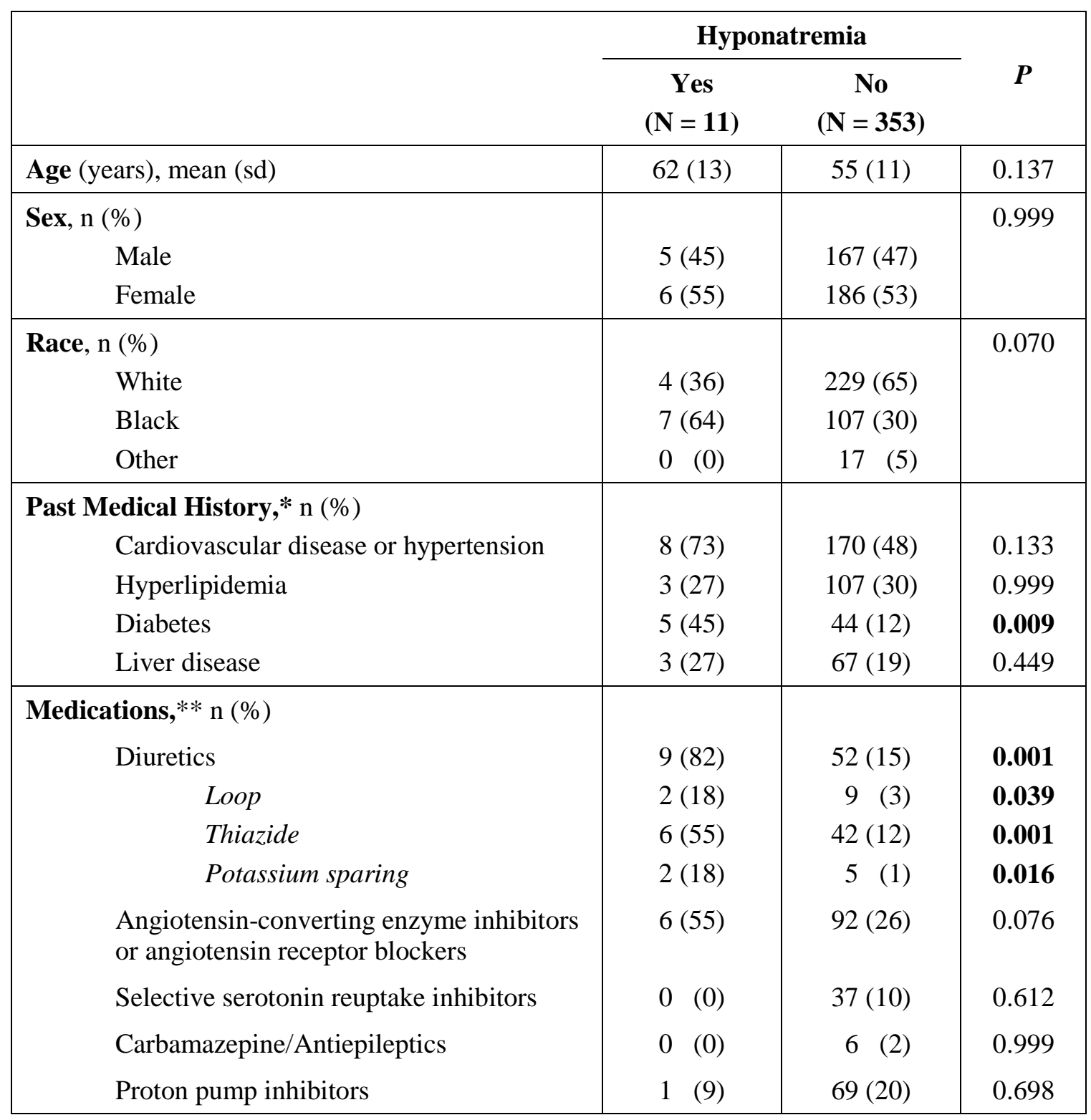

SD: standard deviation.

(*) Multiple medical conditions and medications could be reported. 
Table 3. Number of patients with low electrolyte levels at baseline and on the day of colonoscopy (worst value pre- and post-colonoscopy).

\begin{tabular}{|c|c|c|c|c|}
\hline & $\begin{array}{c}\text { PEG-SD } \\
(\mathrm{N}=180)\end{array}$ & $\begin{array}{c}\text { PEG-ELS } \\
(\mathrm{N}=184)\end{array}$ & $\begin{array}{l}\text { Range of } \\
\text { Abnormal }\end{array}$ & $\boldsymbol{P}$ \\
\hline \multicolumn{5}{|l|}{ Sodium: hyponatremia (<135 mmol/L), n (\%) } \\
\hline Baseline* & -- & -- & & \\
\hline Pre-Colonoscopy* & $5(3)$ & $4(2)$ & & 0.748 \\
\hline Post-Colonoscopy* & $3(2)$ & $3(2)$ & & 0.999 \\
\hline Day of colonoscopy** & $7(4)$ & $4(2)$ & $131-134$ & 0.376 \\
\hline \multicolumn{5}{|l|}{ Potassium: hypokalemia (<3.5 mmol/L), n (\%) } \\
\hline Baseline & $4(2)$ & $6(3)$ & & N/A \\
\hline Day of colonoscopy** & $9(5)$ & $14(8)$ & $2.5-3.4$ & 0.390 \\
\hline \multicolumn{5}{|l|}{ Calcium: hypocalcemia $(<8.5 \mathrm{mg} / \mathrm{dL}), * * * \mathrm{n}(\%)$} \\
\hline Baseline & $0(0)$ & $2(1)$ & & N/A \\
\hline Day of colonoscopy** & $10(6)$ & $17(9)$ & $7.3-8.48$ & 0.230 \\
\hline \multicolumn{5}{|l|}{ Chloride: hypochloremia (<89 mmol/L), n (\%) } \\
\hline Baseline & 0 & 0 & & \\
\hline Day of colonoscopy $* *$ & 0 & 0 & NA & \\
\hline
\end{tabular}

N/A: not applicable (statistical test not meaningful for baseline because of randomization).

(*) No patients had hyponatremia at baseline as this was an exclusion criterion. Pre-colonoscopy $=$ after purgative completion and prior to colonoscopy; post-colonoscopy $=$ after colonoscopy and completion of 1L IV Plasma-lyte. One patient was hyponatremic both pre- and post-colonoscopy and counted once. (**) Day of colonoscopy determination was based on the lowest measured value for each electrolyte on the day of colonoscopy (both pre- and post-colonoscopy).

$(* * *)$ Corrected for albumin. 
Table 4. Change in electrolytes from baseline to day of colonoscopy (worst value pre- or postcolonoscopy).

\begin{tabular}{|l|ccc|ccc|c|}
\hline & \multicolumn{3}{|c|}{ PEG-SD (N= 180) } & \multicolumn{3}{c|}{ PEG-ELS (N = 184) } & P \\
& Baseline & $\begin{array}{c}\text { Day of } \\
\text { Colon* }\end{array}$ & Change & Baseline & $\begin{array}{c}\text { Day of } \\
\text { Colon* }\end{array}$ & Change & for \\
change
\end{tabular}

sd: standard deviation.

(*) Day of Colon: day of colonoscopy value (minimum of pre- and post-colonoscopy value for sodium, potassium, calcium, and chloride, and maximum of pre- and post-colonoscopy value for osmolality).

(**) Calcium corrected for albumin 
Table 5. Preparation completion and side effects, and colonoscopy quality measures and findings.

\begin{tabular}{|c|c|c|c|}
\hline & $\begin{array}{c}\text { PEG-SD } \\
(\mathrm{N}=180)\end{array}$ & $\begin{array}{c}\text { PEG-ELS } \\
(\mathrm{N}=184)\end{array}$ & $\mathbf{P}$ \\
\hline$\geq 90 \%$ preparation completion, $\mathrm{n}(\%)$ & $177(98)$ & $174(95)$ & 0.087 \\
\hline $\begin{array}{l}\text { Fluid intake before procedure }(\mathrm{L}),{ }^{*} \mathrm{n}(\%) \\
\qquad 3 \mathrm{~L} \\
3-5 \mathrm{~L} \\
>5 \mathrm{~L}\end{array}$ & $\begin{array}{c}24(13) \\
145(81) \\
10(6)\end{array}$ & $\begin{array}{l}43(24) \\
120(66) \\
18(10)\end{array}$ & 0.007 \\
\hline $\begin{array}{l}\text { Any side effect, } \mathrm{n}(\%) \\
\text { Nausea } \\
\text { Vomiting } \\
\text { Abdominal pain } \\
\text { Bloating } \\
\text { Light-headedness }\end{array}$ & $\begin{array}{l}110(61) \\
57(32) \\
7 \quad(4) \\
48(27) \\
79(44) \\
25(14)\end{array}$ & $\begin{array}{l}127(69) \\
42(23) \\
12(7) \\
50(27) \\
88(48) \\
31(17)\end{array}$ & $\begin{array}{l}0.124 \\
0.061 \\
0.347 \\
0.999 \\
0.463 \\
0.470\end{array}$ \\
\hline $\begin{array}{l}\text { Beck Anxiety Inventory, } \mathrm{n}(\%) \\
\text { Minimal }(0-7) \\
\text { Mild }(8-15) \\
\text { Moderate }(16-25) \\
\text { Severe }(26-63)\end{array}$ & 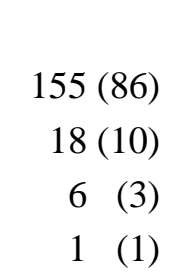 & $\begin{array}{r}155(84) \\
27(15) \\
2 \quad(1) \\
0 \quad(0)\end{array}$ & 0.162 \\
\hline Adequate preparation, whole colon, $* *$ n (\%) & $155(87)$ & $158(86)$ & 0.878 \\
\hline Adequate preparation, right colon, $* * * \mathrm{n}(\%)$ & $150(85)$ & $155(86)$ & 0.882 \\
\hline $\begin{array}{c}\text { Number of adenomas per patient, }{ }^{+} \mathrm{n}(\%) \\
0 \\
1 \\
2 \\
3+\end{array}$ & $\begin{array}{l}122(68) \\
22(12) \\
21(12) \\
14 \quad(8)\end{array}$ & $\begin{array}{l}124(68) \\
29(16) \\
15(8) \\
15(8)\end{array}$ & 0.587 \\
\hline $\begin{array}{l}\text { Any high-risk adenoma or cancer, }{ }^{++} \mathrm{n}(\%) \\
\text { No } \\
\text { Yes }\end{array}$ & $\begin{array}{r}162(91) \\
17(10)\end{array}$ & $\begin{array}{r}174(95) \\
9(5)\end{array}$ & 0.106 \\
\hline
\end{tabular}

(*) Fluid intake unknown for 1 PEG-SD patient and 3 PEG-ELS patients.

(**) Whole-colon preparation quality not applicable for 2 patients (1 in each group) who did not undergo colonoscopy and missing for 1 additional PEG-SD patient.

$(* * *)$ Right-colon preparation quality not applicable for 2 patients (1 in each group) who did not undergo colonoscopy and missing for 4 additional patients ( 2 in each group).

(+) Colonoscopy findings not assessed for 2 patients ( 1 in each group) who did not undergo colonoscopy. $(++)$ Any adenoma $\geq 10 \mathrm{~mm}$ or with high-grade dysplasia or with villous component, or cancer (2 adenocarcinomas detected, both in the PEG-SD group). 
Figure 1. Patient Flow Diagram

Figure 2. Preparation quality.

The primary outcome for preparation quality was adequate (excellent or good) vs. inadequate (fair or poor).-Excellent for whole colon: PEG-SD=30\% vs. PEG-ELS=52\%. Good for whole colon: PEG$\mathrm{SD}=57 \%$ vs. PEG-ELS 34\%, $\mathrm{p}=0.001$. 